\title{
LAPAROSCOPIC SURGERY FOR TREATMENT OF INCISIONAL LUMBAR HERNIA
}

\author{
M. TOBIAS-MACHADO, FREDDY J. RINCON, MARCO T. LASMAR, JOAO P. ZAMBON, \\ ROBERTO V. JULIANO, ERIC R. WROCLAWSKI
}

Section of Urology, ABC Medical School, Santo Andre, Sao Paulo, Brazil

\begin{abstract}
Objective: To present results obtained with laparoscopic correction of incisional lumbar hernia in patients with minimum follow-up of 1 year.

Materials and Methods: We prospectively studied 7 patients diagnosed with incisional lumbar hernia after physical examination and computerized tomography. We used laparoscopic transperitoneal access through 3 ports. One polypropylene mesh was introduced in the abdominal cavity and fixed by titanium clamps to the margins of the hernia ring following release of the peritoneum.

Results: All cases were successfully completed with no conversion required. Mean surgical time was 120 minutes and discharge from hospital occurred between the 1 st and the 2 nd postoperative days. There were no intraoperative complications or hernia recurrence in any case. Postoperatively, we had 2 minor complications: one case of seroma that resolved spontaneously after 60 days and one patient presenting lumbar pain that persisted until the 3 rd postoperative month. The return to usual activities occurred on average 3 weeks following intervention. Of the 7 patients, 6 were satisfied with the esthetical and functional effect produced by the procedure.

Conclusions: The surgical correction of incisional lumbar hernia by laparoscopic access is an excellent option for a minimally invasive treatment, with adequate long-term results.
\end{abstract}

Key words: lumbar region; hernia; surgical procedures, operative; laparoscopy

Int Braz J Urol. 2005; 31: 309-14

\section{INTRODUCTION}

Lumbar hernias are not common, with 2 weak sites existing in the region: the superior (GrynfelttLesshalft) and inferior (Petit) lumbar triangle. All others are known as diffuse lumbar hernias, which are usually associated with conventional extraperitoneal lumbar access (1).

Some surgical repair procedures have been described; the most frequently used being either the open technique with primary closure or the use of prosthetic material. Open surgery requires a large incision and extensive exposure and dissection of the herniated area. Additionally, the margins of the her- nia ring are poorly defined and often require a peritoneal opening for establishing its limits $(2,3)$.

Despite the wide use of the laparoscopic techniques for treating ventral abdominal hernias, a few services have reported sporadic cases using the laparoscopic approach for correction of lumbar defects. Preliminary results suggest that this technique shows advantages concerning patient recovery, especially in shorter hospital stays and prompter returns to normal activities (3-6).

This study aims to present and discuss the long-term results of the laparoscopic repair for incisional lumbar hernias. 


\section{MATERIALS AND METHODS}

\section{Patient Selection and Follow-up}

From January 2002 to January 2004, we prospectively studied 7 patients with incisional lumbar hernias who had undergone previous lumbotomies. Diagnosis was obtained by physical examination, including palpation of the ring's margins, and documented through computerized tomography (Figure1). Patients with any formal contraindication for laparoscopic surgery, coagulation disorders or connective tissue disease were excluded from the study.

Variables pertaining to patients were described and analyzed, such as age, gender, body mass index (BMI), cause of previous lumbar incision such as data relative to the procedure and patient's outcome, such as surgical time, blood loss, analgesic requirements, complications, conversion rate, hospital stay, recovery time until returning to normal activities, and functional and esthetic features.

All included patients were followed up by our outpatient service 7,30, 90, 180 days and finally 1 year following surgery, when a patient satisfaction questionnaire was applied and a control computerized tomography was performed to objectively document the results. The minimal follow-up time for including the results in this study was 1 year.

\section{Surgical Time}

Laparoscopic repair with transperitoneal access was used in all cases. Antibiotic prophylaxis was performed with cefalotin. Patients were placed in right or left lateral decubitus according to the side of herniation and the table was inclined 60 degrees. The first 10-mm Hasson trocar was inserted through the umbilical incision under direct viewing (Figure-2). The cavity was then insufflated through the access trocar with $\mathrm{CO}_{2}$, until a tension of $15 \mathrm{~mm} \mathrm{Hg}$ was reached. Immediately afterwards, the 0 degree optics was introduced and the cavity was inspected to check for the presence of the hernia ring. The herniation area was transilluminated through the peritoneal cavity in order to plan the proper size of the polypropylene mesh (Figure-3).

The second 5-mm port was placed under direct viewing at the mid-clavicular line $2 \mathrm{~cm}$ below the umbilical scar, and the third 12-mm port (suited for the stapler) was placed at the midline between the navel and the xiphoid process (Figure-2).

The peritoneum was released while medially withdrawing the colon that is typically included in the defect in order to expose the entire hernia ring (Figure-4). External palpation of the wall can help to accurately delimitate the defect. The surgical table must allow the patient to be arranged in many different positions for complete dissection of the defect.

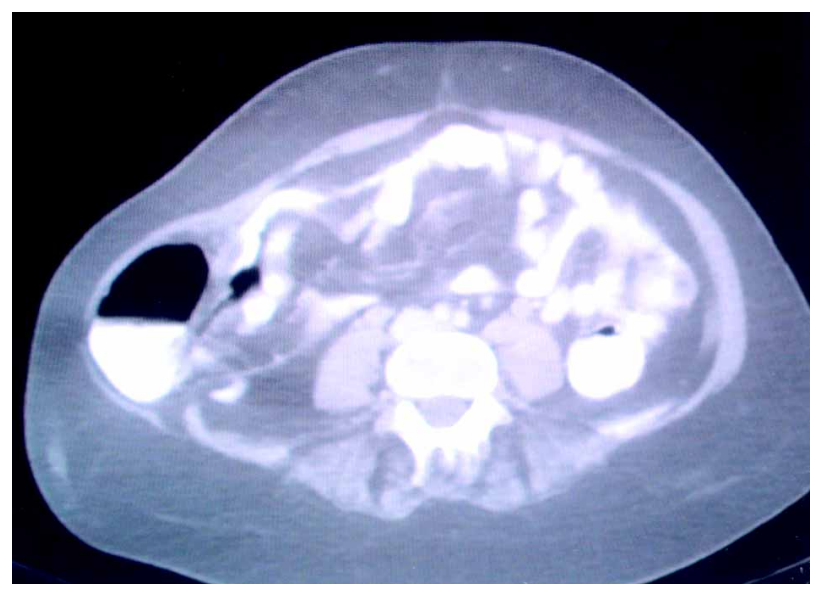

Figure 1 - Computerized tomography showing the abdominal wall defect in the lumbar region.

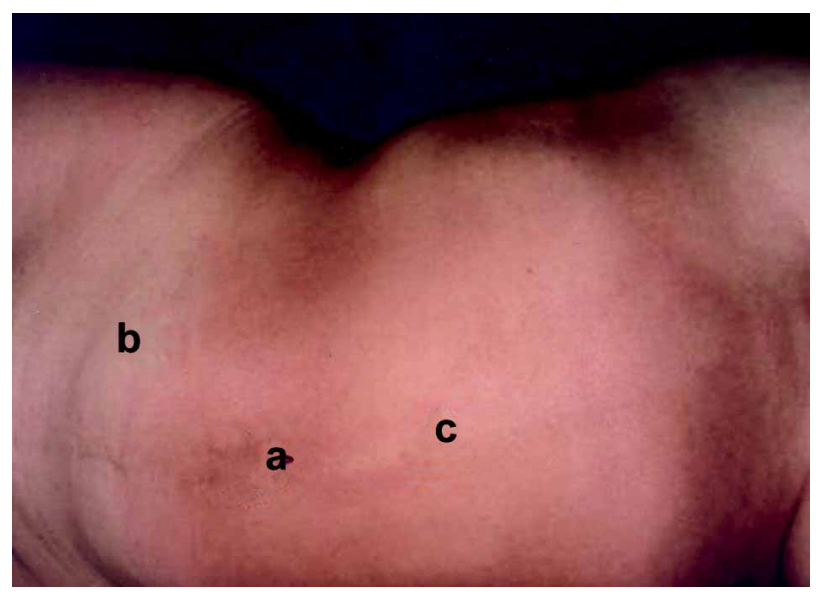

Figure 2 - Position of the ports in the abdominal region for repair of incisional lumbar hernia: $\quad a=10-\mathrm{mm}$ trocar at the umbilicus; $\quad b=5$-mm trocar at mid-clavicular line, $2 \mathrm{~cm}$ below the umbilicus; $c=12-\mathrm{mm}$ trocar at midline between umbilicus and xiphoid process. 
Subsequently, the mesh was inserted into the cavity through the 12-mm trocar and fixed on the wall by an articulate hernia "stapler" using titanium clamps at the margins of the defect (Figure-4). Fixation limits are paravertebral musculature posteriorly, the costal arch superiorly, the iliac spine inferiorly and the abdominal wall musculature anteriorly. During this procedure, $\mathrm{CO}_{2}$ tension was reduced to $7-10 \mathrm{~mm} \mathrm{Hg}$ in order to make the fixation of the mesh easier. Next, the entire mesh was covered by the previously dissected peritoneum and clamped to the wall to prevent it contacting the intestinal loops. Finally, the
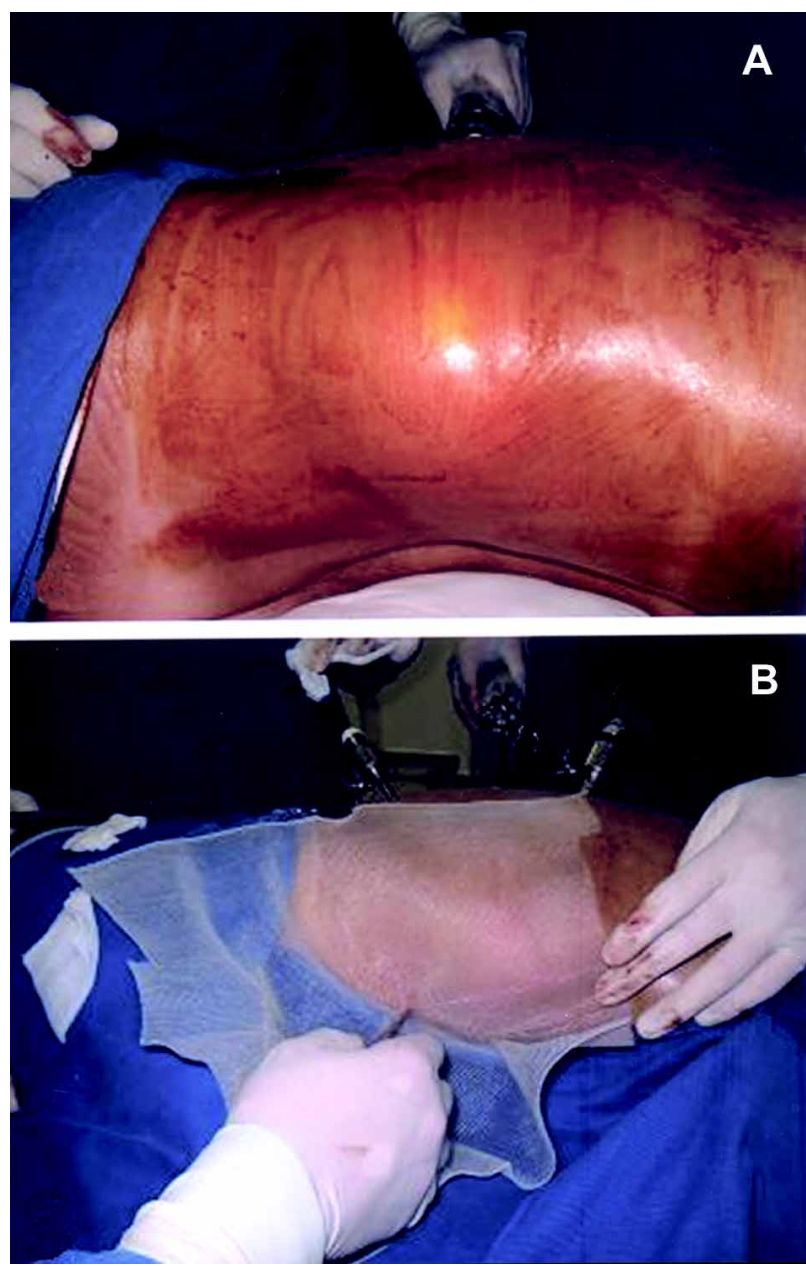

Figure 3 - A) Transillumination of herniation area through the peritoneal cavity. B) Planning of polypropylene mesh size. cavity was reviewed, the ports were removed and the incisions were closed. No drain was left close to the mesh.

\section{RESULTS}

Mean age was 52 years (40 - 65), with BMI from 20-25 (5 cases) and 26-30 (2 cases).

The wall defects ranged in size from $6 \times 8$ $\mathrm{cm}$ to $10 \times 15 \mathrm{~cm}$ (mean $8 \times 12 \mathrm{~cm}$ ).

Three patients were male and 4 were female, with 4 cases occurring on the left side and 3 cases
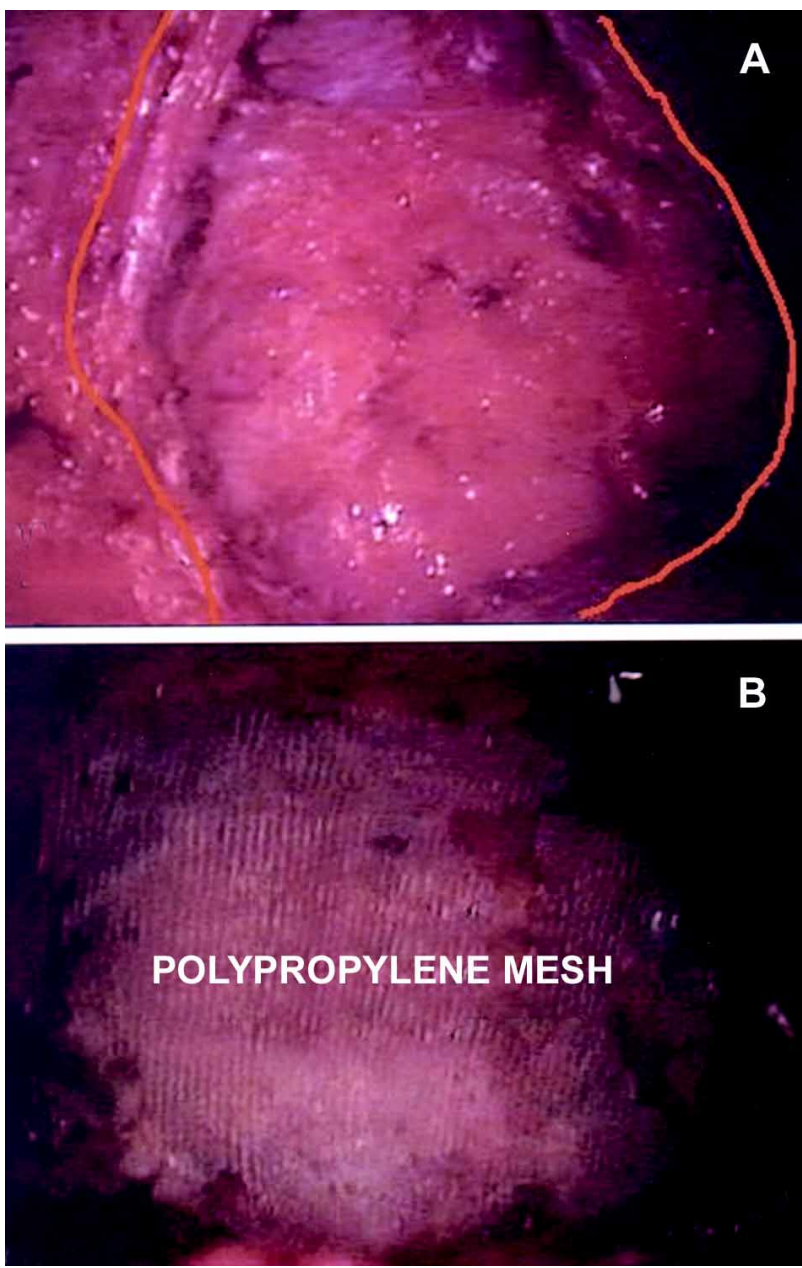

Figure 4-A) Dissected hernia ring. B) Mesh fixed to the wall with titanium clamps on the defect's margins. 
on the right side. In relation to the surgery that caused the previous lumbar incision, there were 3 cases of nephrectomy for kidney donation, 2 cases of nephrectomy due to renal tumor, 1 case of nephrectomy due to hydronephrosis and 1 case of pyelolithotomy.

All procedures were successfully completed by laparoscopic access. During laparoscopic inspection it was possible to distinctively assess the size of the hernia ring and anatomical structures involved in the hernial defect in all patients. The polypropylene mesh was easily inserted into the cavity and fixed by titanium clamps to the ring margins through the 12$\mathrm{mm}$ port.

Surgical time ranged from 90 to 150 minutes (mean 120). There were no intraoperative complications and mean blood loss was $70 \mathrm{~mL}(50$ - 80). Analgesia was obtained using only dipyrone on the first postoperative day in 6 cases. Discharge from hospital occurred on average 12 to 36 hours (mean 24) following surgery. Patients returned to their usual activities 2 to 5 weeks after surgery (mean 3 ).

As far as postoperative complications were concerned, we found 2 minor complications, specifically one case of seroma that resolved spontaneously after 60 days and one female patient presented lumbar pain that lasted until the 3rd postoperative month. This case, which was interpreted as neuropathic pain, required treatment with major analgesics, tricyclic anti-depressants and corticoids for symptom improvement. Probably, a clamp used for fixating the mesh was applied to some nervous bundle at the posterior abdominal wall.

We did not observe a recurrence of hernia in any of the patients during a mean follow-up of 12 months.

The control tomography performed 1 year after the surgery revealed good positioning of the mesh that had been fixed by clamps and repair of the defect in all patients (Figure-5). The esthetic and functional aspect of the defect as reported by the patient was very adequate in 6 out of 7 cases when compared with the preoperative aspect. One patient who presented muscular atonia at the incision's anterior portion before surgery was partly satisfied with the esthetic result.

\section{COMMENTS}

Lumbar hernias are relatively rare, with a little more than 300 cases found in the literature $(1,2,7)$. They can be classified into congenital (10 - 20\%) or acquired $(80-90 \%)$ hernias. Acquired hernias are divided into 2 types - spontaneous and traumatic (incisional) $(1,2,4)$.

The present study included patients with acquired traumatic lumbar hernias secondary to lumbar incision for conventional renal surgery. Though the classical lumbotomy is still largely used in our country, the increasing use of laparoscopic and percutaneous surgery for treating surgical conditions of the kidneys and adrenal glands will certainly reduce the occurrence of such complications.

In general, lumbar hernias are diagnosed using clinical criteria (6). The main complaint of patient is the perception of a reducible tumor with solid consistency in the incision area, which can be accompanied by lumbar discomfort. Recent publications describe the importance of computerized tomography to identify the hernia, demonstrating in detail the anatomy and differential diagnosis with other pathologies $(1,6,8)$. The computerized tomography was an important diagnostic method for identifying, confirming and objectively documenting the hernia in this study.

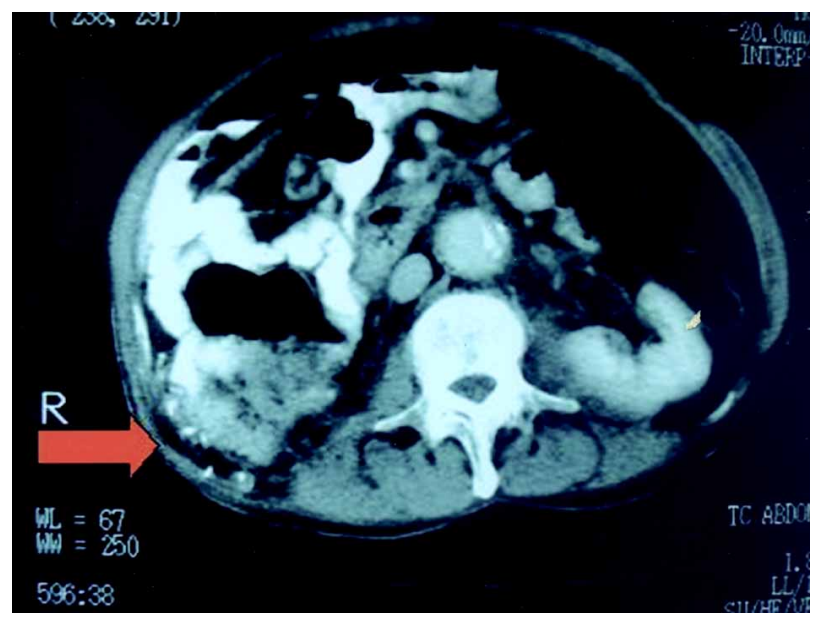

Figure 5 - Control tomography performed 1 year after surgery evidenced good positioning of the clamp-fixed mesh and repair of hernial defect. 
When untreated, lumbar hernia can reach gigantic proportions, thus increasing the risk of incarceration $(25 \%)$ and strangulation $(8 \%)(6)$. The hernial content can include the epiploon, small or large bowel, spleen and the kidney itself (1).

If permitted by the patient's general condition, the lumbar hernia always has surgical indication with several techniques being described in the literature. Due to its rarity, there is no standardized technique. The difficulty in delimiting the margins of the fascial defect, the weakness of the involved structures, the participation of a bone element, and the surgeon's expertise are all elements taken into account during surgical planning $(1,6)$.

The open technique for reconstruction of lumbar hernias requires a large incision, which is often associated with more severe pain, a longer convalescence period and increased morbidity $(1,4)$. For the conventional repair of such hernias, natural structures from the region itself or synthetic materials (made of polypropylene or polytetrafluoroethylene) can be used. Results described for surgery without mesh have been poor, probably due to the low tensile quality of the local tissues, which is why the repair with synthetic material has been preferred $(1,4,5)$.

With the intention of reducing the morbidity observed with the conventional technique while maintaining the results from open surgery with mesh, the laparoscopic access has been recently described.

Using the expertise in repair of ventral hernias that has been accumulated in many centers, the same principles could be applied to lumbar hernias as well. Initial experiences have shown significant advantages of the laparoscopic approach over conventional surgery. The majority of studies describing this technique has reported low morbidity, less significant pain and earlier returns to normal activities $(2,4,6)$. Other studies have confirmed that this access promotes optimal visualization of the ring's limits, is safe and simple, and is considered a minimally invasive procedure $(2,3,9)$.

The repair of lumbar hernia by laparoscopic approach was first published in 1997 by Heniford et al. (7). The following year, Arca et al. (4) published the first results from experience with 7 patients with lumbar hernias treated by the laparoscopic approach.
The authors concluded that there was improved visualization of the anatomical defects, reduced hospital stay, and no recurrence in this sample during a 15month period.

In the present study, we observed an excellent exposure of structures and achieved perfect anatomical visualization of the hernia ring. There was little postoperative pain, reduced mean hospital stay, and the return to usual activities occurred promptly. During the 12-month follow-up period, no recurrence of herniation was evidenced. In one case, the posterior hernial defect was repaired, but patient satisfaction was not completely achieved due to atonia of the abdominal wall secondary to a nervous lesion occurring after the lumbotomy.

Among the small number of published studies on laparoscopic repair of lumbar hernias, none of them has described significant complications (2-7). Comparative studies between the open and laparoscopic approach reported in the literature refer only to the surgical treatment of ventral incisional hernias. There are no such studies involving lumbar herniation, which, in a certain way, does not allow us to definitely conclude which access is best $(10,11)$. Our impression, however, is that the laparoscopic repair seems to have advantages concerning the visualization of the hernial defect and the postoperative recovery.

\section{CONCLUSIONS}

The laparoscopic repair of incisional lumbar hernia is a minimally invasive procedure with moderate complexity, which promotes adequate functional and esthetic results. It provides excellent exposure and definition of the wall defect limits, mild postoperative pain, short hospital stay and early return to normal activities. If comparative studies confirm the superiority of the laparoscopic approach in relation to the open technique, the laparoscopic procedure could become the method of choice for repair of lumbar hernias.

\section{REFERENCES}

1. Moreno-Egea A, Torralba-Martinez JA, Morales G, Fernandez T, Girela E, Aguayo-Albasini JL: Open vs 
laparoscopic repair of secondary lumbar hernias: a prospective nonrandomized study. Surg Endosc. 2005; 19: 184-7.

2. Sakarya A, Aydede H, Erhan MY, Kara E, Ilkgul O, Yavuz C: Laparoscopic repair of acquired lumbar hernia. Surg Endosc. 2003; 17: 1494.

3. Maeda K, Kanehira E, Shino H, Yamamura K: Laparoscopic tension-free hernioplasty for lumbar hernia. Surg Endosc. 2003; 17: 1497.

4. Arca MJ, Heniford BT, Pokorny R, Wilson MA, Mayes J, Gagner M: Laparoscopic repair of lumbar hernias. J Am Coll Surg. 1998; 187: 147-52.

5. Bickel A, Haj M, Eitan A: Laparoscopic management of lumbar hernia. Surg Endosc. 1997; 11: 112930 .

6. Shekarriz B, Graziottin TM, Gholami S, Lu HF, Yamada H, Duh QY, et al.: Transperitoneal preperitoneal laparoscopic lumbar incisional hernorrhaphy. J Urol. 2001; 166: 1267-9.

7. Heniford BT, Iannitti DA, Gagner M: Laparoscopic inferior and superior lumbar hernia repair. Arch Surg. 1997; 132: 1141-4.

8. Baker ME, Weinerth JL, Andriani RT, Cohan RH, Dunnick NR: Lumbar hernia: diagnosis by CT. AJR Am J Roentgenol. 1987; 148: 565-7.

9. Parker HH 3rd, Nottingham JM, Bynoe RP, Yost MJ: Laparoscopic repair of large incisional hernias. Am Surg. 2002; 68: 530-3; discussion 533-4.

10. Chari R, Chari V, Eisenstat M, Chung R: A case controlled study of laparoscopic incisional hernia repair. Surg Endosc. 2000; 14: 117-9.

11. Goodney PP, Birkmeyer CM, Birkmeyer JD: Short-term outcomes of laparoscopic and open ventral hernia repair: a meta-analysis. Arch Surg. 2002; 137: 1161-5.

Received: February 24, 2005

Accepted after revision: April 29, 2005
Correspondence address:

Dr. Marcos Tobias-Machado

Rua Graúna, 104 / 131

São Paulo, SP, 04514-000, Brazil

E-mail: tobias-machado@uol.com.br 